\title{
Mandibular advancement and obstructive sleep apnoea: a method for determining effective mandibular protrusion
}

\author{
L.C. Dort*, E. Hadjuk\# and J.E. Remmers
}

ABSTRACT: The objectives of the study were to test the hypotheses that it is possible, during routine polysomnography (PSG), to prospectively identify favourable candidates for mandibular repositioning appliance (MRA) therapy in the treatment of obstructive sleep apnoea (OSA) and to accurately estimate an optimal protrusive distance at which to fabricate the MRA.

A series of subjects underwent a remotely controlled mandibular positioner (RCMP) test during PSG monitoring. The ability of the RCMP test to eliminate OSA and the target protrusion at which that occurred was compared with the success of a custom oral MRA in the 33 subjects who completed the protocol.

The RCMP test was a success in 15 subjects and a failure in 18 subjects. Appliance therapy was initiated in 38 subjects and completed in 33. MRA therapy was successful at target protrusion in $80 \%$ of subjects who had a successful RCMP test and failed in $78 \%$ of those who failed the RCMP test.

In conclusion the remotely controlled mandibular positioner test outcome demonstrated a statistically significant association with mandibular repositioning appliance outcome. The target protrusion determined during the remotely controlled mandibular positioner test was the effective therapeutic protrusion in subjects with a successful remotely controlled mandibular positioner test.

KEYWORDS: Mandibular advancement device, mandibular repositioning device, polysomnography, sleep apnoea diagnosis and treatment, treatment outcome, treatment success

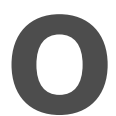
bstructive sleep apnoea (OSA) is a condition that affects $4-9 \%$ of adults [1-4]. OSA is a risk factor for hypertension and consequent cardiovascular morbidity, as well as excessive daytime sleepiness, and it's associated impairment in function and quality of life [5]. Nasal continuous positive airway pressure (CPAP) is the standard treatment for this disorder, but compliance can be problematic [6-9]. The rate of refusal to begin or continue CPAP in the first week varies $5-50 \%$ [10]. A further $12-$ $25 \%$ of subjects are expected to discontinue CPAP within 3 yrs [10]. Side-effects and problems of CPAP that affect compliance include stuffy or dry nasal passages, mask irritation, awakenings, machine noise, claustrophobia and gastric distention. Oral appliances (OAs) are an alternative to CPAP therapy. Crossover studies comparing OAs and CPAP [11-15] have generally shown OAs to be less effective (15-55\% therapeutic success) than CPAP. Patients have reportedly preferred OA therapy to CPAP [12-15]. Trials comparing $\mathrm{OA}$ therapy to no treatment or inactive controls have been shown to be effective in treating $45-70 \%$ of patients with OSA. It is likely that OAs are perceived as being less intrusive and less disruptive to personal life than CPAP [16-19]. Mandibular repositioning appliances (MRA) are a category of promising OAs used to treat OSA. In this approach, the mandible is repositioned ventrally and inferiorly by the appliance, thereby enhancing pharyngeal patency [20]. Mandibular protrusion has shown to have a "dose-dependent" effect on the pharyngeal cross-sectional area [21].

Adjustable MRAs allow progressive protrusion of the mandible under clinical supervision. Determining the effective protrusive setting for an appliance usually takes place over several weeks and may involve multiple visits to the dental provider's office. This long regimen delays the implementation of effective therapy, which may increase the cost of therapy and decrease compliance.

Identifying patients who are likely to be successful MRA candidates would be desirable from both therapeutic and financial perspectives.

\section{AFFILIATIONS}

${ }^{*}$ Dept of Surgery, University of Calgary,

\#Alberta Lung Association Sleep Centre, Foothills Hospital, and "Division of Respiratory Medicine, Dept of Medicine, University of Calgary, AB, Canada.

CORRESPONDENCE

L.C. Dort

2716

7th Ave NW

Calgary

$\mathrm{AB}$

T2N 1 A7

Canada

Fax: 14032503055

E-mail: dort@ucalgary.ca

Received

June 282004

Accepted after revision:

December 022005

SUPPORT STATEMENT

This study was supported by the Alberta Heritage Foundation for Medical Research. 
Clinical predictors of MRA treatment outcome have been evaluated in a number of studies. Females, who are not supine dependent and males with supine-dependent OSA are more likely to achieve therapeutic success with MRAs [22]. Improved treatment response has been associated with lower values of age, body mass index (BMI), neck size and respiratory disturbance index (RDI) [23-25]. Cephalometric features have been shown to be associated with treatment response. These include, but are not limited to: anteroposterior oropharynx dimension, overjet, mandible length, and mandibular plane-to-hyoid distance [23, 26]. Imaging studies, using magnetic resonance imaging of the pharynx, suggest an association between lack of occlusion during the Mueller manoeuvre and MRA treatment success [27]. The data from these studies investigating clinical predictors of MRA success are too limited to formulate general recommendations.

The present study addresses the need for a tool that prospectively identifies favourable candidates for MRA therapy, ideally prior to involving a dentist, and accurately estimates optimal protrusive distance as useful therapeutic information for the treating dentist. A recent study [28] examined a tool to protrude the mandible during polysomnography (PSG) and evaluate the effect on OSA. In this pilot study of 19 compliant subjects, the remotely controlled mandibular positioner (RCMP) demonstrated positive and negative predictive values of 90 and $89 \%$, respectively, in predicting MRA treatment success. The purpose of the present study was threefold: 1) to confirm the predictive capabilities of the RCMP; 2) to investigate the association between the RCMP effective protrusive measurement and MRA success; and 3) to evaluate the effectiveness of the RCMP when employed by standard PSG personnel. The current authors hypothesised that when the RCMP test was efficacious, the protrusive measurement $(\mathrm{mm})$ at which this occurred would also be effective with an MRA [28].

\section{METHODS}

Patients were recruited from Alberta Lung Association Sleep Centre (Calgary, AB, Canada), which is the major referral centre for southern Alberta with a population of $>1.5$ million [29]. Referral sources for the centre include family physicians, interns, otolaryngologists, psychiatrists and dentists. As standard management, patients with mild-to-moderate OSA $\left(\right.$ RDI $<30$ events $\cdot h^{-1}$ ) may be offered a referral for a consultation regarding oral appliance therapy. Patients received a home diagnostic study [30], and patients with OSA were informed of the therapeutic options. Those who chose to pursue oral appliance therapy were approached to participate in the current study. Those with suitable dentition underwent baseline clinical evaluation.

An overnight PSG, using an RCMP (www.sagatech.ca., figs 1 and 2), was performed on all subjects. A polysomnographic technician performed the overnight RCMP trial. On the night of the study, the technician fabricated a custom temporary trial appliance using pre-formed trays customised with impression material. The vertical opening at the central incisors was $\sim 2-4 \mathrm{~mm}$, with the trial appliance in position in centric occlusion (normal closing) position.

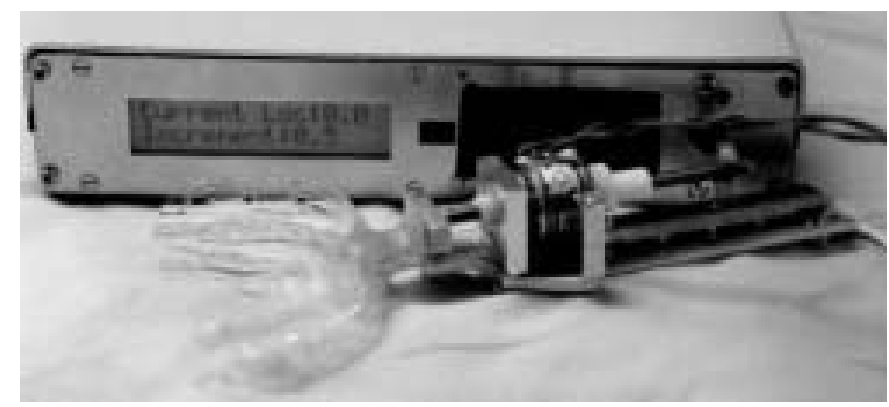

FIGURE 1. A remotely controlled mandibular positioner device.

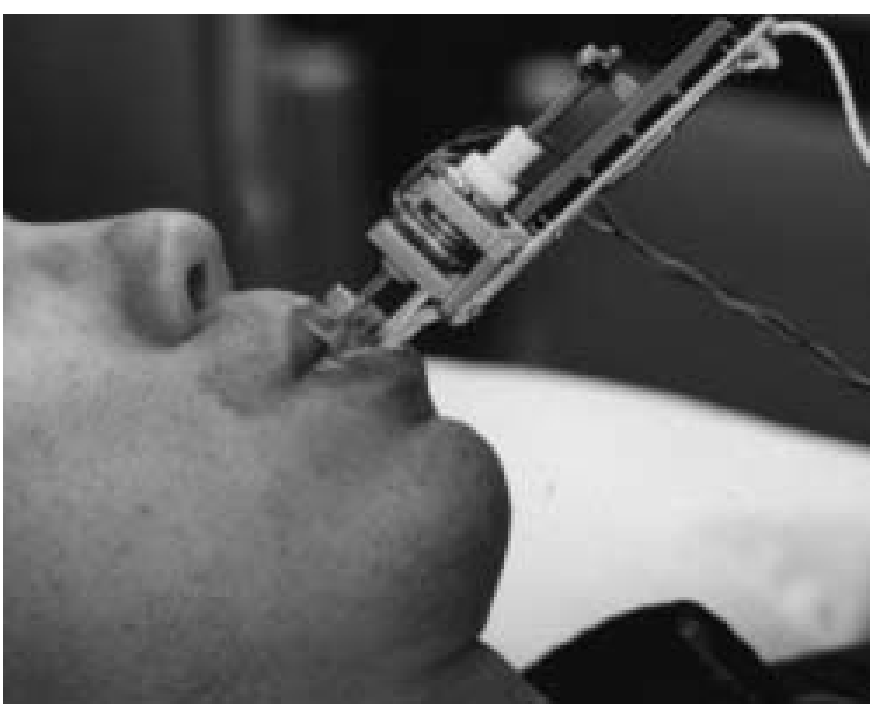

FIGURE 2. A remotely controlled mandibular positioner device placed intraorally without polysomnography set-up.

A stepper motor, which was attached to a strut extending from the lower tray, applied force to the upper tray. The motor was remotely controlled using a computer interface program. The force exerted by an axial foot upon the labial surface of the maxillary tray advanced the mandible. The protrusive distance from the initial position was quantified using linear potentiometer feedback.

A computerised polysomnographic system (Sandman, Nellcor Puriton Bennett; Melvin Ltd, Ottawa, ON, Canada) recorded all PSG data. The standard montage consisted of: ECG, electrooculogram, electroencephalogram (EEG, C4/A1), submental electromyogram and digitally recorded snoring sounds. Bilateral nares pressure was recorded by catheters anchored on the upper lip and connected to a pressure transducer that qualitatively assessed airflow. Inductance plethysmography (Respitrace, Ambulatory monitoring, Ardsley, NY, USA) detected thoracic-abdominal motion. Oximetry (Model 3700; Ohmeda, Denver, CO, USA) was used to determine arterial oxygen saturation $\left(\mathrm{Sa}_{2} \mathrm{O}_{2}\right)$.

The mandible was advanced in 1-mm increments following documentation of baseline obstructive respiratory events in stable stage 2 sleep. An obstructive event was defined either as 
an apnoea (absence of airflow for $10 \mathrm{~s}$ ) or as a hypopnoea (a $>10 \mathrm{~s}$ reduction in either airflow or thoracoabdominal movement, associated with a $>3 \%$ drop in $\mathrm{Sa}_{1} \mathrm{O}_{2}$ and increased pressure deflections in PSG). If an EEG arousal occurred, no further advancement was attempted until stable sleep (at least three epochs of sleep without arousals) returned. If the patient awoke and was unable to fall asleep within $10 \mathrm{~min}$, all advancement was reversed and re-initiated following the appearance of stable stage 2 sleep. Mandibular advancement continued until elimination of obstructive apnoeas, hypopnoeas and nocturnal oxygen desaturation was achieved.

The RCMP test results were scored as follows: successful if obstructive events and snoring were eliminated; failure if the patient could not tolerate the RCMP, if there were technical difficulties with the RCMP device or obstructive events and snoring remained in non-rapid eye movement (REM) or REM sleep. A dentist (L.C. Dort, Alberta Lung Association Sleep Centre) who was blinded to the results of the RCMP study fitted the subjects with an MRA. The MRA Klearway ${ }^{\mathrm{TM}}$ [17] used in this trial is a custom fabricated MRA that is individually titratable to determine the most desirable protrusion over an 11-mm range. The dentist was given a target protrusive distance for all subjects, regardless of the outcome of the RCMP study. When the RCMP test was considered a success, the protrusive distance at which it occurred was reported to the dentist. When the RCMP test failed, protrusive distance of two-thirds of the subject's protrusive capabilities was reported to the dentist. The twothirds protrusive distance is the routine initial treatment position for the Klearway ${ }^{\mathrm{TM}}$ [17].

The MRA was fabricated at target protrusion. When a patient reported the ability to wear the appliance for a minimum of $4 \mathrm{~h}$ at a time at target protrusion during sleep, home portable monitoring was repeated. The MRA was fixed at target protrusion prior to monitoring.

MRA therapy was considered successful if the RDI was reduced to $<15 \cdot h^{-1}$ and $<30 \%$ of the baseline. If portable monitoring showed the RDI to be $<15 \cdot \mathrm{h}^{-1}$, no further advancement was made to the MRA, unless the patient wished to reduce snoring as reported by the bed partner. If the RDI at target was $>15 \cdot \mathrm{h}^{-1}$, the MRA was advanced until the patient's discomfort prevented further protrusion. This is referred to as final adjustment. At that time, a second post-treatment portable monitoring was performed. Patients completed a baseline and post-treatment Epworth Sleepiness Scale (ESS) [31], and the Calgary Sleep Apnea Quality of Life Index questionnaires [32].

The RDI was assessed at baseline, target and final position using a portable monitor that recorded $\mathrm{Sa}_{2} \mathrm{O}_{2}$, heart rate, snoring sounds and body position. An off-line algorithm analysed the $\mathrm{Sa}_{1} \mathrm{O}_{2}$ signal to determine RDI. The monitor's diagnostic performance characteristics for OSA provided a close estimate of the PSG-determined apnoea/hypopnoea index (AHI) [30], with a sensitivity and specificity of 98 and $88 \%$, respectively, at an AHI diagnostic criterion of $>15 \cdot \mathrm{h}^{-1}$.

Descriptive summary statistical analysis was performed. Nonnormally distributed variables were geometrically transformed. Paired t-tests compared baseline RDI with RDI and the MRA at target protrusion. Two-by-two frequency tables, using Fisher's exact test, were employed to examine the association between RCMP test and MRA outcome, as well as to calculate sensitivity, specificity, and positive and negative predictive values. Target RDI was used as the dependent variable in a multiple regression analysis of clinical predictors. Baseline average RDI, baseline supine RDI, age, BMI, neck circumference and ESS were employed as the independent variables.

The Conjoint Ethics Committee of the University of Calgary (Calgary, AB, Canada) approved the study.

\section{RESULTS}

A total of 44 patients were recruited for the study, on the basis of the initial ambulatory sleep study. During the PSG RCMP study, six subjects did not meet the inclusion criteria of RDI $>5$. The RCMP test and MRA construction was completed for 38 subjects. Five subjects were lost to follow-up after MRA fitting. The study was completed in the remaining 33 subjects. The study population comprised predominately middle-aged, overweight males. The mean \pm SD initial RDI was $26.9 \pm 18.3$ (table 1).

MRAs were fitted for 38 subjects. Five subjects did not respond to follow-up contact attempts. Baseline characteristics of those who were lost to follow-up did not differ significantly from those who completed the study. The RCMP test was a success in 15 out of $33(45 \%)$ and failed in 18 out of $33(55 \%)$ subjects. Five subjects were failures because they could not tolerate the RCMP device long enough for sufficient data to be recorded. Two subjects had insufficient data due to a lack of REM sleep and there were two technical failures when the RCMP device stopped working part way through the night. The remaining nine subjects tolerated the device but obstructive events were not eliminated.

A significant association $(p=0.001)$ was observed between the RCMP test outcome and the treatment outcome with the MRA at the determined target protrusion. MRA therapy was successful in 12 out of $15(80 \%)$ subjects, who had a successful RCMP test and failed in 14 out of $18(78 \%)$ subjects when the

\section{TABLE 1 Baseline characteristics of the study population}

Characteristics

$\begin{array}{lc}\text { Age yrs } & 45.0 \pm 9.9 \\ \text { Females } & 2(0.06) \\ \mathbf{B M I} \mathbf{~ k g} \cdot \mathbf{m}^{-\mathbf{2}} & 29.9 \pm 6.3 \\ \mathbf{R D I} \text { events } \cdot \mathbf{h}^{-\mathbf{1}} & 26.9 \pm 18.3 \\ \mathbf{R D I} \text { supine events } \cdot \mathbf{h}^{-1} & 28.13 \pm 27.7 \\ \text { Neck circumference } \mathbf{~ c m} & 41.2 \pm 3.4 \\ \mathbf{S a}_{\mathbf{O}} \mathbf{O}_{\mathbf{2}} & 91.8 \pm 2.6 \\ \mathbf{\%} \text { of time } \mathbf{O}_{\mathbf{2}}<\mathbf{9 0} \% & 18.3 \pm 22.1 \\ \text { ESS } & 8.0 \pm 4.0 \\ \text { Baseline Calgary SAQLI } & 4.3 \pm 1.2\end{array}$

Data are presented as mean $\pm S D$ and $n(\%)$. BMI: body mass index; RDI: respiratory disturbance index; $\mathrm{Sa}_{1} \mathrm{O}_{2}$ : arterial oxygen saturation; ESS: Epworth Sleepiness Scale; SAQLI: Sleep Apnea Quality of Life Index. 
RCMP test was a failure (table 2). The sensitivity and specificity of the RCMP test were 75 and $82 \%$, respectively.

The individual changes in RDI with MRA treatment at target protrusion are grouped by RCMP test outcome category and are illustrated in figures 3 and 4 . Paired t-tests showed a significant RDI change $(\mathrm{p}<0.0002)$ only in the subjects with a successful RCMP test. Additional MRA treatment success was attained in three out of $18(17 \%)$ subjects of those who failed the RCMP test when advancement was continued beyond target. Further advancement in the group with successful RCMP tests did not increase MRA effectiveness.

The mean target advancement was $6.3 \mathrm{~mm}$ in the group where the RCMP was a therapeutic success and $4.8 \mathrm{~mm}$ in the group that failed the RCMP test. The maximum protrusion mean was $8.6 \mathrm{~mm}$. This was the mean of the demonstrable mandibular protrusion measured clinically at the time the appliances were fabricated for both groups. There were no significant differences in baseline characteristics between RCMP successes and failures (table 3).

A multiple regression analysis revealed the baseline average RDI as the only significant determinant $(p<0.002)$ of target RDI. Target RDI did not depend on baseline average RDI, baseline supine RDI, age, BMI, neck circumference or ESS. Further regression analysis showed no significant dependence of percentage change in RDI on any of these characteristics.

\section{DISCUSSION}

An RCMP test evaluated efficacy of mandibular advancement in controlling OSA during PSG and determined therapeutic protrusive distance (target) when possible in 38 subjects. Custom MRAs were fitted for all subjects. The MRA compliance rate was 33 out of $38(87 \%)$. MRA therapy achieved therapeutic success at target in $12 / 15$ (80\%) subjects who had a successful RCMP test. MRA therapy failed therapeutically at target in 14 out of $18(78 \%)$ subjects whose RCMP test was a failure. The overall success in treating OSA with the MRA at target in compliant subjects was 16 out of $33(49 \%)$. Follow-up evaluation with MRAs at target protrusion demonstrated a sensitivity and specificity of 75 and $82 \%$, respectively, in determining MRA treatment outcome. A previously published pilot study reported a sensitivity and specificity of 90 and $89 \%$,

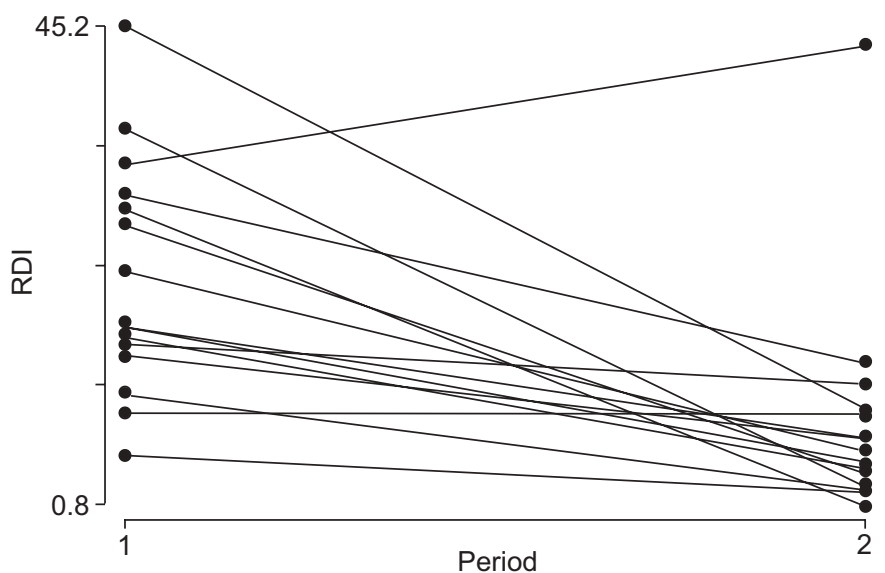

FIGURE 3. Change in respiratory disturbance index (RDI) for group one for remotely controlled mandibular positioner success.

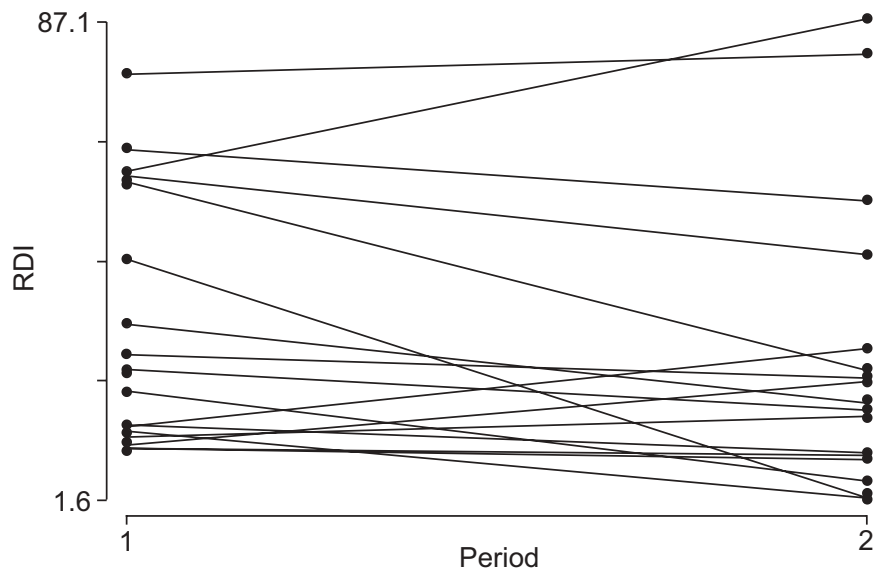

FIGURE 4. Change in respiratory disturbance index (RDI) for group two for remotely controlled mandibular positioner failure.

respectively. Baseline characteristics of both studies show no significant differences. Five subjects in the present study could not tolerate the RCMP device. None of the subjects in the initial study were intolerant of the RCMP. The difference in tolerance

\section{TABLE 2} Association between remotely controlled mandibular positioner (RCMP) test and mandibular repositioning appliance (MRA) outcome at target protrusion using different criteria ${ }^{\#}$

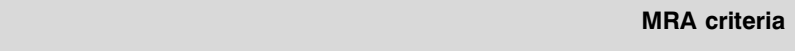

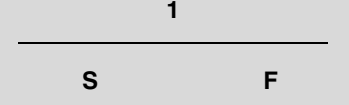

RCMP

$S$

Fisher's exact

F

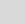

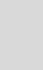

12
4
0.001

\footnotetext{
S: success; F: failure; RDI: respiratory disturbance index. ${ }^{*}: 1$ : RDI decrease of $\geqslant 30 \%$ and $R D I<15 \cdot h^{-1} ; 2: R D I$ decrease of $\geqslant 50 \%$ and $R D I<15 \cdot h^{-1} ; 3:$ RDI decrease of $\geqslant 30 \%$ and $\mathrm{RDI}<10 \cdot \mathrm{h}^{-1} ; 4$ : RDI decrease of $\geqslant 50 \%$ and $\mathrm{RDI}<10 \cdot \mathrm{h}^{-1}$.
} 


\begin{tabular}{|c|c|c|c|}
\hline \multirow{3}{*}{\multicolumn{2}{|c|}{$\begin{array}{l}\text { TABLE } 3 \\
\text { Characteristic }\end{array}$}} & $\begin{array}{l}\text { y remotely } \\
\text { oner (RCN }\end{array}$ & d \\
\hline & & \multicolumn{2}{|c|}{ RCMP } \\
\hline & & Success & Failure \\
\hline \multicolumn{2}{|l|}{ Age yrs } & $42.7 \pm 8.7$ & $47.6 \pm 11.4$ \\
\hline \multicolumn{2}{|l|}{ BMI $\mathbf{k g} \cdot \mathbf{m}^{-2}$} & $29.3 \pm 3.9$ & $30.7 \pm 8.2$ \\
\hline \multicolumn{2}{|c|}{ RDI events $\cdot h^{-1}$} & $21.8 \pm 11.0$ & $32.3 \pm 22.2$ \\
\hline \multicolumn{2}{|c|}{ RDI supine events $\cdot h^{-1}$} & $28.1 \pm 39.6$ & $28.1 \pm 22.1$ \\
\hline \multicolumn{2}{|c|}{ Neck circcumference $\mathrm{cm}$} & $41.1 \pm 2.3$ & $41.7 \pm 4.4$ \\
\hline \multicolumn{2}{|l|}{$\mathrm{Sa}, \mathrm{O}_{2} \%$} & $92.5 \pm 1.9$ & $90.8 \pm 2.9$ \\
\hline \multicolumn{2}{|c|}{$\%$ of time $\mathrm{O}_{2}<90 \%$} & $13.1 \pm 14.4$ & $25.8 \pm 27.3$ \\
\hline \multicolumn{2}{|l|}{ ESS } & $9.3 \pm 3.9$ & $6.7 \pm 3.7$ \\
\hline \multicolumn{2}{|c|}{ Baseline Calgary SAQLI } & $4.4 \pm 1.0$ & $4.3 \pm 1.4$ \\
\hline
\end{tabular}

may be explained by the protocol of the current study, which was designed to evaluate the RCMP effectiveness under standard PSG conditions. A PSG technician trained in the RCMP technique, yet with limited experience with oral devices and conditions, set up the RCMP. While test tolerance may have been improved by using dental health professionals, the clinical utility of the test is reduced by the need for the presence of these professionals in the PSG setting.

The success of the MRA may have been confounded by the absence of supine-dependent OSA in the predominantly male subjects evaluated. MARKLUND et al. [22] demonstrated increased success with MRAs in males with supine-dependent OSA.

Target protrusion as determined by the RCMP test was predictive of MRA therapeutic protrusion in all those who had a successful RCMP test. In three of the subjects who failed the RCMP test, advancement beyond two-thirds of the demonstrable protrusive distance on the night of the RCMP eventually resulted in success with the MRA. These three subjects tolerated the RCMP test but continued to have events at the maximum protrusion determined on the night of the RCMP test. This was the maximum protrusion they were able to demonstrate immediately preceding the RCMP test. The eventual therapeutic success with the MRA, at protrusion greater than the originally measured maximum, may reflect accommodation to the MRA over time or technical error at the time of the RCMP test. A dental health professional in attendance at the RCMP test might improve the test reliability. This restriction would significantly reduce the clinical utility of the test.

The negative predictive capability of the RCMP test could be of potential clinical value in presenting treatment options, i.e. patients with a negative or equivocal test could be informed that chances of success with MRA treatment would be poor. Such information may, in turn, enhance acceptance of CPAP therapy. Patients with an RCMP test predicting a successful outcome would possibly benefit by eliminating both time to effective treatment and decreasing cost. The current authors were unable to identify any characteristics, such as baseline RDI or BMI, which could help categorise potential successes and failures. The success of MRA therapy without consideration of RCMP success/failure grouping ranged 49-56\%, depending on the criteria used to determine success. A randomised control trial comparing Klearway ${ }^{\mathrm{TM}}$ to CPAP determined therapeutic efficacy to be $61-80 \%$, depending on the severity of the OSA [17]. A pilot RCMP trial indicated effectiveness in the range of $53 \%$ [28].

A multiple regression analysis examining baseline characteristics did not show baseline RDI, age, weight, mean $\mathrm{Sa}_{1} \mathrm{O}_{2}$ or ESS to be predictive of per cent reduction in RDI with the MRA. A model formulated by MEHTA et al. [25], which includes both clinical features and cephalometric features, predicts that $82 \%$ of the variation in the RDI after MRA therapy is accounted for by neck circumference, baseline RDI and two cephalometric measurements, i.e. retropalatal airway space and the angle between anterior cranial base and mandibular plane. While statistically a good fit, the clinical usefulness of this model has not been tested. Prospective studies are required to confirm that this model will aid clinicians in predicting MRA outcome after baseline sleep test and cephalometric analysis. Recent prospective case series of oral appliances have reported success ranging $54-81 \%$ in reducing RDI by $\geqslant 50 \%$ [33]. The model of MEHTA et al. [25], which combines clinical and cephalometric features, does not significantly improve the potential to predict success with MRAs compared with the range of reported success in reducing the RDI by $\geqslant 50 \%$ [33].

Two recent, small studies have investigated titration of MRAs during sleep. One of these included six subjects using the MRA during PSG [34]. The patient was awakened, the custom appliance removed and advanced during the sleep study. At the end of the PSG with the custom appliance, all candidates could have been classified as successes, i.e. RDI with MRA $\leqslant 15$. A limitation of this case series is that subjects were selected who had had a custom appliance fabricated and had undergone an adjustment period. This was not a predictive study.

The second study involved seven subjects [35]. A PSG titration study with a trial appliance was compared to a PSG study with a custom appliance. The RDI was reduced to $<10 \cdot \mathrm{h}^{-1}$ in $42.9 \%$ of subjects. The success with this group of subjects was similar to that reported by other published studies. In addition, the mean \pm SD mandibular advancement was $12.6 \pm 2.7 \mathrm{~mm}$. This advancement is considerably greater than the mean \pm SD target protrusion of $5.6 \pm 2.3 \mathrm{~mm}$ and the mean $\pm \mathrm{SD}$ maximum protrusion of $8.6 \pm 3.4 \mathrm{~mm}$ determined by the present study. Most patients would be unlikely to tolerate an advancement of $12.6 \mathrm{~mm}$ in the long term. Although this study was aimed at predicting a therapeutic effect, the subjects had not been adapted to the custom appliance. The second PSG at the therapeutic advancement was conducted on the first night the subject had the custom appliance. It is possible that the subjects would not have been able to adapt to the appliance.

The target distance determined by the RCMP provided a reliable estimate of therapeutic advancement. Determination of target advancement has potential in increasing MRA 
compliance, decreasing the titration time and decreasing the need for extensive titration capabilities of an appliance. Even when the MRA was well tolerated and the RCMP test was successful the MRA was not effective in treating OSA in all subjects. Three of the 15 patients, who were predicted to have been treated successfully with MRA were failures. The RDI of one subject increased with the MRA and the RDI changed negligibly for the other two subjects. These failures may be related to environmental factors in the home, such as allergies, alcohol and body position. While the increase in area of the upper airway in response to mandibular advancement has been shown to be dependent on the amount of advancement [21], it is also variable. The response of the anteroposterior diameter of the velopharynx has been shown to enlarge in those who respond to MRA therapy [36].

The results of an RCMP test could significantly influence future MRA design and treatment when used in conjunction with other predictive factors. A therapeutic target and prediction of success would eliminate the sometimes long titration period, which is a probable compliance inhibitor with appliance therapy. A nonadjustable design set at target could decrease the time necessary for titration and thereby reduce cost.

The ability to prospectively identify candidates who will experience therapeutic success continues to limit the clinical utility of mandibular repositioning appliances. The remotely controlled mandibular positioner test can aid identification of candidates but additional factors must be investigated and added to the remotely controlled mandibular positioner predictive capabilities to give subjects $>80 \%$ probability of therapeutic success with a mandibular repositioning appliance.

\section{REFERENCES}

1 Young T, Palta M, Dempsey J, et al. The occurrence of sleep-disordered breathing among middle-aged adults. $N$ Engl J Med 1993; 328: 1230-1235.

2 Olson LG, King MT, Hensley MJ, Saunders NA. A community study of snoring and sleep-disordered breathing. Prevalence. Am J Respir Crit Care Med 1995; 152: 711-716.

3 Gislason T, Almqvist M, Eriksson G, Taube A, Boman G. Prevalence of sleep apnea syndrome among Swedish men--an epidemiological study. J Clin Epidemiol 1988; 41: 571-576.

4 Bearpark H, Elliott L, Grunstein R. Snoring and sleep apnea. A population study in Australian men. Am J Respir Crit Care Med 2004; 151: 1459-1465.

5 Peppard PE, Young T, Palta M, et al. Prospective study of the association between sleep-disordered breathing and hypertension. N Engl J Med 2000; 342: 1378-1384.

6 Chervin RD, Theut S, Bassetti C, Skatrud J, Aldrich MS. Compliance with nasal CPAP can be improved by simple interventions. Sleep 1997; 20: 284-289.

7 Meurice JC, Dore P, Paquereau J, et al. Predictive factors of long-term compliance with nasal continuous positive airway pressure treatment in sleep apnea syndrome. Chest 1994; 105: 429-433.

8 Pepin JL, Krieger J, Rodenstein D, et al. Effective compliance during the first 3 months of continuous positive airway pressure. A European prospective study of 121 patients. Am J Respir Crit Care Med 1999; 160: 1124-1129.

9 Reeves-Hoche MK, Meck R, Zwillich CW. Nasal CPAP: an objective evaluation of patient compliance. Am J Respir Crit Care Med 1994; 149: 149-154.

10 Engleman HM, Wild MR. Improving CPAP use by patients with the sleep apnoea/hypopnoea syndrome (SAHS). Sleep Med Rev 2003; 7: 81-99.

11 Clark GT, Blumenfeld I, Yoffe N, Peled E, Lavie P. A crossover study comparing the efficacy of continuous positive airway pressure with anterior mandibular positioning devices on patients with obstructive sleep apnea. Chest 1996; 109: 1477-1483.

12 Ferguson KA, Ono T, Lowe AA, keenan SP, Fleetham JA. A randomized crossover study of an oral appliance $v s$ nasalcontinuous positive airway pressure in the treatment of mild-moderate obstructive sleep apnea. Chest 1996; 109: 1269-1275.

13 Ferguson KA, Ono T, Lowe AA, al-Majed S, Love LL, Fleetham JA. A short-term controlled trial of an adjustable oral appliance for the treatment of mild to moderate obstructive sleep apnoea. Thorax 1997; 52: 362-368.

14 Randerath WJ, Heise M, Hinz R, Ruehle KH. An individually adjustable oral appliance vs continuous positive airway pressure in mild-to-moderate obstructive sleep apnea syndrome. Chest 2002; 122: 569-575.

15 Tan YK, L'Estrange PR, Luo YM, et al. Mandibular advancement splints and continuous positive airway pressure in patients with obstructive sleep apnoea: a randomized cross-over trial. Eur J Orthod 2002; 24: 239-249.

16 Krieger J. New frontiers in the management of obstructive sleep apnoea syndrome. Monaldi Arch Chest Dis 1998; 53: 515-519.

17 Lowe AA, Sjoholm TT, Ryan CF, Fleetham JA, Ferguson KA, Remmers JE. Treatment, airway and compliance effects of a titratable oral appliance. Sleep 2000; 23: Suppl. 4, S172-S178.

18 Schmidt-Nowara W, Lowe A, Wiegand L, Cartwright R, Perez-Guerra F, Menn S. Oral appliances for the treatment of snoring and obstructive sleep apnea: a review. Sleep 1995; 18: 501-510.

19 Cistulli PA, Gotsopoulos H, Marklund M, Lowe AA. Treatment of snoring and obstructive sleep apnea with mandibular repositioning appliances. Sleep Med Rev 2004; 8: 443-457.

20 Isono S, Tanaka A, Sho Y, Konno A, Nishino T. Advancement of the mandible improves velopharyngeal airway patency. J Appl Physiol 1995; 79: 2132-2138.

21 Kato J, Isono S, Tanaka A, et al. Dose-dependent effects of mandibular advancement on pharyngeal mechanics and nocturnal oxygenation in patients with sleep-disordered breathing. Chest 2000; 117: 1065-1072.

22 Marklund M, Persson M, Franklin KA. Treatment success with a mandibular advancement device is related to supine-dependent sleep apnea. Chest 1998; 114: 1630-1635.

23 Liu Y, Zeng X, Fu M, Huang X, Lowe AA. Effects of a mandibular repositioner on obstructive sleep apnea. Am J Orthod Dentofacial Orthop 2000; 118: 248-256.

24 Cartwright RD. Predicting response to the tongue retaining device for sleep apnea syndrome. Arch Otolaryngol 1985; 111: 385-388. 
25 Mehta A, Qian J, Petocz P, Darendeliler MA, Cistulli PA. A randomized, controlled study of a mandibular advancement splint for obstructive sleep apnea. Am J Respir Crit Care Med 2001; 163: 1457-1461.

26 Eveloff SE, Rosenberg CL, Carlisle CC, Millman RP. Efficacy of a Herbst mandibular advancement device in obstructive sleep apnea. Am J Respir Crit Care Med 1994; 149: 905-909.

27 Sanner BM, Heise M, Knoben B, et al. MRI of the pharynx and treatment efficacy of a mandibular advancement device in obstructive sleep apnoea syndrome. Eur Respir J 2002; 20: 143-150.

28 Tsai WH, Vazquez JC, Oshima T, et al. Remotely controlled mandibular positioner predicts efficacy of oral appliances in sleep apnea. Am J Respir Crit Care Med 2004; 170: 366-370.

292003 Official Population List. Alberta, Alberta Municipal Affairs, Edmonton, 2003.

30 Vazquez JC, Tsai WH, Flemons WW, et al. Automated analysis of digital oximetry in the diagnosis of obstructive sleep apnoea. Thorax 2000; 55: 302-307.
31 Johns MW. A new method for measuring daytime sleepiness: The Epworth Sleepiness Scale. Sleep 2004; 14: 540-545.

32 Flemons WW, Reimer MA. Development of a diseasespecific health-related quality of life questionnaire for sleep apnea. Am J Respir Crit Care Med 1998; 158: 494-503.

33 Ferguson KA. The role of oral appliance therapy in the treatment of obstructive sleep apnea. Clin Chest Med 2003; 24: 355-364.

34 Raphaelson MA, Alpher EJ, Bakker KW, Perlstrom JR. Oral appliance therapy for obstructive sleep apnea syndrome: progressive mandibular advancement during polysomnography. Cranio 1998; 16: 44-50.

35 Petelle B, Vincent G, Gagnadoux F, Rakotonanahary D, Meyer B, Fleury B. One-night mandibular advancement titration for obstructive sleep apnea syndrome: A pilot study. Am J Respir Crit Care Med 2002; 165: 1150-1153.

36 Tsuiki S, Lowe AA, Almeida FR, Fleetham JA. Effects of an anteriorly titrated mandibular position on awake airway and obstructive sleep apnea severity. Am J Orthod Dentofacial Orthop 2004; 125: 548-555. 